\title{
Partially Solvable Pair-Coupling Models with Seniority-Conserving Interactions
}

\author{
D. J. Rowe \\ Department of Physics, University of Toronto, Toronto, Ontario M5S 1A7, Canada \\ G. Rosensteel \\ Department of Physics, Tulane University, New Orleans, Louisiana 70118
}

(Received 29 December 2000; published 5 October 2001)

\begin{abstract}
Given a single $j$-shell Hamiltonian, the algebraic conditions for conservation of seniority are derived from a quasispin tensor decomposition of the two-nucleon interaction. This makes it possible to construct useful solvable and partially solvable shell-model Hamiltonians with eigenstates classified by a spectrum generating algebra. Applications are made to the low-lying energy levels of the $N=50$ nuclear isotones.
\end{abstract}

DOI: $10.1103 /$ PhysRevLett.87.172501

A model is said to be solvable if its energy levels can be determined analytically and partially solvable if some of its energy levels can be determined analytically [1]. Solvable and partially solvable models are important in both physics and mathematics. Besides their direct use to explain physical phenomena, such models are valuable in physics for testing approximate many-body theories. In mathematics, they provide a framework for studying related systems of solvable and partially solvable differential equations.

A model may be solvable, for example, because its eigenstates are identified completely by the quantum numbers of a subgroup chain. Likewise a model may be partially solvable because a set of quantum numbers uniquely defines a subset of so-called multiplicity free states. Models described by Alhassid and Leviatan [2] as having a partial dynamical symmetry are of the latter type. The possibility of deriving the energies of general multiplicityfree states by (hopefully simple) algebraic methods poses an interesting and significant challenge.

The models we consider have physical applications in nuclear physics to singly closed shell nuclei. It is understood that, for such nuclei, the $J=0$ pairing interaction is the dominant component of the nuclear interaction. For many shell model calculations, it is appropriate therefore to adopt pair-coupled basis states in which the pairing interaction is diagonal; such basis states are classified by seniority quantum numbers [3]. Evidence for the dominance of the pairing interaction in nuclei is provided, for example, by the low-energy spectra of even closed-shell-plus-two nuclei such as shown for ${ }^{92} \mathrm{Mo}$ in Fig. 1 . The ${ }^{92} \mathrm{Mo}$ spectrum shows that the $J=0$ two-nucleon state is lowered more than the $J \neq 0$ states. However, although smaller, the interactions in the $J=2$ and other $J \neq 0$ pair states are non-negligible. For two-nucleon states, $J \neq 0$ interactions are easily included. But, for many particles they mix states of different seniority and destroy the simplicity of the algebraically solvable pair-coupling model.

In fact, it is known from the work of Racah in atomic spectroscopy [4] that seniority remains a good quantum number for any two-body interaction acting within a $j$ sub-
PACS numbers: 21.60.Cs, 02.20.-a, 21.30.Fe, 31.15.-p

shell when $j \leq 7 / 2$. We show that seniority is also conserved for a wide range of two-body interactions for larger values of $j$. Within a single $j$ shell, there are $(2 j+1) / 2$ linearly independent two-body interactions. We find that linearly independent combinations of these interactions can be found such that all but one conserve seniority for $9 / 2 \leq j \leq 13 / 2$ and all but two conserve seniority for $15 / 2 \leq j \leq 19 / 2$. In general, the interactions that do not conserve seniority are a small fraction of the total. This makes it possible to construct realistic solvable and partially solvable single-shell models.

In the context of a single- $j$ shell model, states are described as multiplicity free if they are uniquely defined by seniority and angular momentum quantum numbers. For example, seniority-two states are multiplicity free as are the states of largest angular momentum, $J=J_{\max }$, and next largest, $J=J_{\max }-2$, of any seniority. The existence of multiplicity-free states suggests that the Schrödinger equation for a seniority-conserving Hamiltonian in a single- $j$ shell should be partially solvable. This turns out to be the case. However, while analytic expressions may exist for multiplicity-free states, it does not appear to be easy to determine them, in general. We give here expressions for the seniority-two states and the states of $J=J_{\max }$ and $J_{\max }-2$ of any seniority.

Energy levels for all states can be computed numerically by diagonalization of the Hamiltonian when $j$ is not too large. Such calculations are facilitated by use of the representation theory of the unitary symplectic algebra, $\mathrm{USp}(2 j+1)[7,8]$. This is discussed briefly below but determination of the matrix elements of the $\operatorname{USp}(2 j+1)$ irreps, in the physical angular momentum basis, is deferred to a following paper.

The SU(2) quasispin algebra for nucleons of a single type in a single $j$ shell is spanned by the operators

$$
\begin{aligned}
& \hat{S}_{+}=\sum_{m=1 / 2}^{j} a_{j m}^{\dagger} a_{j \bar{m}}^{\dagger}, \quad \hat{S}_{-}=\sum_{m=1 / 2}^{j} a^{j \bar{m}} a^{j m}, \\
& \hat{S}_{0}=\frac{1}{4} \sum_{m=-j}^{j}\left(a_{j m}^{\dagger} a^{j m}-a^{j m} a_{j m}^{\dagger}\right)=\frac{1}{2}(\hat{n}-\Omega),
\end{aligned}
$$



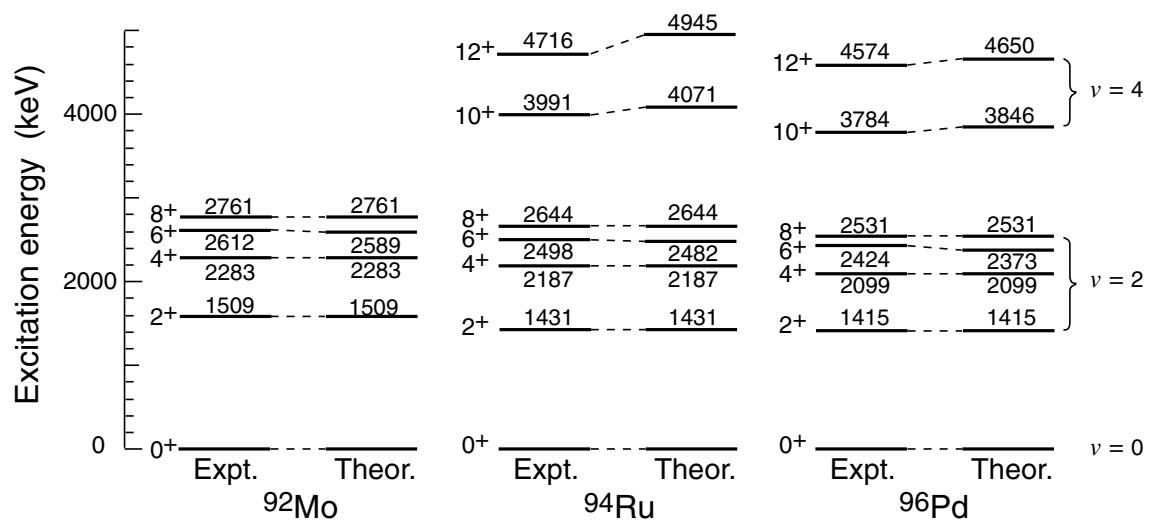

FIG. 1. Low-energy spectra of $N=50$ isotones fitted with a seniority conserving interaction for $1 g_{9 / 2}$-shell configurations. where $\left\{a_{j m}^{\dagger}, a^{j m}\right\}$ are creation and annihilation operators for a single nucleon (e.g., neutron) satisfying the fermion anticommutation relations

$$
\left\{a^{j m}, a_{j n}^{\dagger}\right\}=\delta_{m n},
$$

and

$$
a_{j \bar{m}}^{\dagger}=(-1)^{j-m} a_{j,-m}^{\dagger}, \quad a^{j \bar{m}}=(-1)^{j-m} a^{j,-m}=a_{j m} ;
$$

$\hat{n}=\sum_{m} a_{j m}^{\dagger} a^{j m}$ is the nucleon number operator and $\Omega=$ $\frac{1}{2}(2 j+1)$. Note that the annihilation operator $a^{j m}$ with superscripts is the Hermitian adjoint of the creation operator $a_{j m}^{\dagger}$. The operator $a_{j m}$ with subscripts is the $m$ component of a spherical tensor. The quasispin operators satisfy the usual $\mathrm{SU}(2)$ commutation relations

$$
\left[\hat{S}_{+}, \hat{S}_{-}\right]=2 \hat{S}_{0}, \quad\left[\hat{S}_{0}, \hat{S}_{ \pm}\right]= \pm \hat{S}_{ \pm} .
$$

States of a single $j$ shell can now be classified by their $\operatorname{SU}(2)$ quasispin $\left(S, S_{0}\right)$ and angular momentum $(J, M)$ quantum numbers. The quasispin quantum numbers are related to seniority $v$ (the number of unpaired particles) and the number $n$ of particles by

$$
S=\frac{1}{2}(\Omega-v), \quad S_{0}=\frac{1}{2}(n-v) .
$$

The SU(2) quasispin algebra can also be used to classify the second quantized operators of a $j$ shell as quasispin tensors [6]. The fermion operators transform into one another as components of a quasispin $S=1 / 2$ tensor;

$$
\begin{aligned}
{\left[\hat{S}_{+}, a_{j m}\right] } & =a_{j m}^{\dagger}, & {\left[\hat{S}_{-}, a_{j m}^{\dagger}\right] } & =a_{j m}, \\
{\left[\hat{S}_{0}, a_{j m}^{\dagger}\right] } & =\frac{1}{2} a_{j m}^{\dagger}, & {\left[\hat{S}_{0}, a_{j m}\right] } & =-\frac{1}{2} a_{j m} .
\end{aligned}
$$

For even $J$, the pair operators $\left\{\hat{A}_{J M}, \hat{C}_{J M}, \hat{B}_{J M}\right\}$, defined by

$$
\begin{aligned}
\hat{A}_{J M} & =\frac{1}{\sqrt{2}}\left(a_{j}^{\dagger} \otimes a_{j}^{\dagger}\right)_{J M}=\frac{1}{\sqrt{2}} \sum_{m n}(j m, j n \mid J M) a_{j n}^{\dagger} a_{j m}^{\dagger} \\
\hat{C}_{J M} & =\left(a_{j}^{\dagger} \otimes a_{j}\right)_{J M}, \quad J \neq 0, \\
\hat{C}_{0} & =\frac{1}{2}\left[\left(a_{j}^{\dagger} \otimes a_{j}\right)_{0}+\left(a_{j} \otimes a_{j}^{\dagger}\right)_{0}\right]=\sqrt{\frac{2}{\Omega}} \hat{S}_{0}, \\
\hat{B}_{J M} & =\frac{1}{\sqrt{2}}\left(a_{j} \otimes a_{j}\right)_{J M},
\end{aligned}
$$

are the $(1,0,-1)$ components of a quasispin $S=1$ tensor and the operators $\left\{\hat{C}_{J M} ; J\right.$ odd $\}$ are quasispin scalars [5].

A two-body interaction, acting within a $(j)^{n}$ configuration space, has the standard expansion

$$
V=-\frac{1}{4} \sum_{J}[J] V^{J}\left[\hat{A}_{J} \otimes \hat{B}_{J}\right]_{0},
$$

where $[J]=\sqrt{2 J+1}$ and

$$
V^{J}=\left\langle j^{2} ; J M|V| j^{2} ; J M\right\rangle .
$$

The $\left[\hat{A}_{J} \otimes \hat{B}_{J}\right]_{0}$ operators are linear combinations of the operators

$X^{0}(J)=\left[\hat{A}_{J} \otimes \hat{B}_{J}\right]_{0}-\left[\hat{C}_{J} \otimes \hat{C}_{J}\right]_{0}+\left[\hat{B}_{J} \otimes \hat{A}_{J}\right]_{0}$,

$X_{0}^{1}(J)=\left[\hat{A}_{J} \otimes \hat{B}_{J}\right]_{0}-\left[\hat{B}_{J} \otimes \hat{A}_{J}\right]_{0}$,

$X_{0}^{2}(J)=\left[\hat{A}_{J} \otimes \hat{B}_{J}\right]_{0}+2\left[\hat{C}_{J} \otimes \hat{C}_{J}\right]_{0}+\left[\hat{B}_{J} \otimes \hat{A}_{J}\right]_{0}$,

where $X^{0}(J)$ is a scalar, $X^{1}(J)$ a vector, and $X^{2}(J)$ a rank-two quasispin tensor. However, the $X_{0}^{S}(J)$ operators are not all linearly independent and most of those that are do not mix seniority. For example, the vector operators

$$
X_{0}^{1}(J)=\left[\hat{B}_{J}, \hat{A}_{J}\right]_{0}=\frac{[J]}{\Omega}(\hat{n}-\Omega),
$$

are all proportional to one another and diagonal in any basis of well-defined nucleon number.

The relationship between the $\left[\hat{A}_{J} \otimes \hat{B}_{J}\right]_{0}$ and $X_{0}^{S}(J)$ operators becomes explicit if the operators $\left[\hat{B}_{J} \otimes \hat{A}_{J}\right]_{0}$ and $\left[\hat{C}_{J} \otimes \hat{C}_{J}\right]_{0}$ are normal ordered by means of the relationships

$$
\begin{array}{rr}
{\left[\hat{B}_{J} \otimes \hat{A}_{J}\right]_{0}=\left[\hat{A}_{J} \otimes \hat{B}_{J}\right]_{0}+\frac{[J]}{\Omega}(\hat{n}-\Omega),} & \text { for } J=0, \\
{\left[\hat{C}_{J} \otimes \hat{C}_{J}\right]_{0}=\left\{\begin{array}{lr}
\frac{1}{2 \Omega}(\hat{n}-\Omega)^{2} & \text { for } J \neq 0, \\
\sum_{\gamma} M_{J \gamma}^{\Omega}\left[\hat{A}_{\gamma} \otimes \hat{B}_{\gamma}\right]_{0}+\frac{[J]}{2 \Omega} \hat{n}, &
\end{array}\right.}
\end{array}
$$

where

$$
M_{J \gamma}^{\Omega}=2[J][\gamma] W(j j j j ; \gamma J),
$$

and $\gamma$ runs over the even integer values from 0 to $(2 j-1)$. It follows that, to within constants and terms linear in the 
number operator,

$$
\begin{gathered}
\sum_{\gamma}\left[M_{J \gamma}^{\Omega}-2 \delta_{J \gamma}\right]\left[\hat{A}_{\gamma} \otimes \hat{B}_{\gamma}\right]_{0}=-X^{0}(J)+\cdots, \\
\sum_{\gamma}\left[M_{J \gamma}^{\Omega}+\delta_{J \gamma}\right]\left[\hat{A}_{\gamma} \otimes \hat{B}_{\gamma}\right]_{0}=X^{2}(J)+\cdots .
\end{gathered}
$$

Claim. - The eigenvalues of the matrix $M^{\Omega}$ are all equal to -1 or 2 . Let $p_{-1}$ and $p_{2}$ denote the number of eigenvalues having values -1 and 2 , respectively. Then, to within constants and terms linear in the number operator, $p_{-1}$ linearly independent combinations of the $2 \Omega$ operators $\left\{\left[\hat{A}_{J} \otimes \hat{B}_{J}\right]_{0} ; J=0, \ldots,(2 j-1)\right\}$ are quasispin scalars, and $p_{2}$ linearly independent combinations are rank-two quasispin tensors.

Proof.-There are $\Omega=(2 j+1) / 2$ linearly independent two-nucleon interactions. Consider the linear combinations of $\left[\hat{A}_{\gamma} \otimes \hat{B}_{\gamma}\right]_{0}$ operators which are eigenvectors of $M^{\Omega}$. It follows from Eqs. (15) and (16) that, to within constants and terms linear in the number operator, the eigenvectors with eigenvalues not equal to 2 are quasispin scalars, and those with eigenvalues not equal to -1 are components of rank-two tensors. The implication is that every eigenvalue of the matrix $M^{\Omega}$ is either equal to 2 or -1 . Diagonalization of $M^{\Omega}$ confirms that this is indeed the case. Therefore $p_{-1}+p_{2}=\Omega$ and there are $p_{-1}$ linearly independent quasispin scalars and $p_{2}$ linearly independent rank-two tensors.

Now observe that the operator $X_{0}^{2}(J=0)$ is a combination of quasispin operators $\left\{\hat{S}_{ \pm}, \hat{S}_{0}\right\}$ and so, even though it is a component of an $S=2$ quasispin tensor, it does not mix states of different seniority. Linear combinations of the remaining $p_{2}-1$ linearly independent $S=2$ operators will, in general, mix seniority. This means that a two-body interaction must satisfy $p_{2}-1$ constraint conditions in order that it should conserve seniority.

Diagonalization of the matrix $M^{\Omega}$ confirms that, for $j \leq$ $7 / 2$, all two-body interactions conserve seniority and that for $9 / 2 \leq j \leq 13 / 2$ there is only one constraint condition; for $15 / 2 \leq j \leq 19 / 2$, there are two constraint conditions. Thus, within a single $j$ shell, seniority is a good quantum number for a wide range of interactions. The eigenvectors of the matrix $M^{\Omega}$ show that, to conserve seniority, the two-body matrix elements, defined by Eq. (8), must satisfy the following linear relationships (computed using Maple with exact arithmetic):

$$
\begin{gathered}
\text { for } j=9 / 2, \quad 65 V^{2}-315 V^{4}+403 V^{6}-153 V^{8}=0, \\
\text { for } j=11 / 2, \quad 1020 V^{2}-3519 V^{4}+637 V^{6}+4403 V^{8}-2541 V^{10}=0, \\
\text { for } j=13 / 2, \quad 1615 V^{2}-4275 V^{4}-1456 V^{6}+3196 V^{8}+5145 V^{10}-4225 V^{12}=0 .
\end{gathered}
$$

The first of these equations was derived previously by Talmi [3] as the condition that the seniority-mixing matrix element of the Hamiltonian should vanish between seniority-one and three states of a $(j=9 / 2)^{3}$ configuration. It is now seen that this condition and similar conditions for $j=11 / 2$ and $j=13 / 2$ are sufficient to ensure that seniority is conserved for all of the corresponding single-shell states.

Energy eigenvalues for the above seniority-conserving models are obtained by the following theorem:

Theorem (Talmi [3]). - Any Hamiltonian with seniority-conserving two-body interactions acting within a single $j$ shell can be expressed in the form

$$
H=\varepsilon \hat{n}-G \hat{S}_{+} \hat{S}_{-}+V_{0},
$$

where $V_{0}$ is a linear combination of the $\left\{\left[\hat{C}_{J} \otimes \hat{C}_{J}\right]_{0} ; J=\right.$ $1,3,5, \ldots\}$ operators.

The utility of this theorem arises from the fact that it expresses the Hamiltonian in terms of the infinitesimal generators $\left\{\hat{S}_{ \pm}, \hat{S}_{0}\right\}$ of the $\mathrm{SU}(2)$ quasispin group and those of the group $\mathrm{USp}(2 j+1)$ of seniority-conserving one-body unitary transformations. The latter, being the quasispin scalar operators $\left\{\hat{C}_{J M}=\left(a^{\dagger} \otimes a\right)_{J M} ; J=1\right.$, $3,5 \ldots, 2 j\}$, commute with elements of the $\mathrm{SU}(2)$ quasispin algebra. Moreover, it is known [8] that the sum of the Casimir invariants of $\mathrm{USp}(2 j+1)$ and $\mathrm{SU}(2)$ takes a constant value throughout the Hilbert space of the single $j$ shell model. This means that the irreps of $\operatorname{USp}(2 j+1)$ are paired with unique irreps of SU(2) and vice versa. It also means that the states of a given particle number and seniority span a $\mathrm{USp}(2 j+1)$ irrep. In the language of Howe [9], $\mathrm{USp}(2 j+1)$ and the $\mathrm{SU}(2)$ quasispin group form a so-called dual pair.

One linear combination of the $\left[\hat{C}_{J} \otimes \hat{C}_{J}\right]_{0}$ operators is the second-order Casimir invariant of $\operatorname{USp}(2 j+1)$ which, as noted above, is linearly related to the Casimir invariant $\hat{S} \cdot \hat{S}$ of the SU(2) quasispin algebra. Another is the Casimir invariant, $\hat{J} \cdot \hat{J} \propto\left[\hat{C}_{1} \otimes \hat{C}_{1}\right]_{0}$, of $\operatorname{SU}(2)_{j}$. Since, the number $p_{1}$ of linearly independent scalars is equal to three for $j=7 / 2$ and $j=9 / 2$, it follows that, one additional linearly independent scalar is needed for $j=$ $7 / 2$ or $9 / 2$; it can be taken as $\left[\hat{C}_{3} \otimes \hat{C}_{3}\right]_{0}$. For $j=$ $11 / 2$, a second operator, e.g., $\left[\hat{C}_{5} \otimes \hat{C}_{5}\right]_{0}$, is needed and, for $j=13 / 2$, a third operator is needed, etc.

The Casimir invariant $\hat{S} \cdot \hat{S}$ of the quasispin algebra has values $s(s+1)$ given in terms of seniority by

$$
4 s(s+1)=j(j+3)+\frac{5}{4}-v(2 j+3-v) .
$$

It follows that, if the eigenstates of the Hamiltonian (20) are labeled $\{|N \rho v J M\rangle\}$, where $N$ is the nucleon number and $\rho$ a multiplicity index, then the corresponding excitation energies are independent of $N$. For example, for $j=9 / 2$ and $N$ even, they are given by 


$$
E_{N \rho v J}=E_{N 0}+a J(J+1)+b v(2 j+3-v)+c Z_{\rho v J},
$$

where $Z_{\rho v J}$ is an eigenvalue of $\left(\hat{C}_{3} \otimes \hat{C}_{3}\right)_{0} / \sqrt{7}$.

By computer calculations (cf. following paper for details) the eigenvalues of $\left(\hat{C}_{3} \otimes \hat{C}_{3}\right)_{0} / \sqrt{7}$ for the multiplicity-free states of $v=2$ are determined to be

$$
\begin{aligned}
Z_{2 J}= & -\frac{4 J(J+1)}{(x+4)(x+3)(x+2)(x+1) x(x-1)(x-2)} \\
& \times\left\{6 x^{4}+24 x^{3}-3[5 J(J+1)+4] x^{2}-6[5 J(J+1)+12] x+2\left[5 J^{2}(J+1)^{2}+20 J(J+1)+12\right]\right\},
\end{aligned}
$$

where $x=2 j$. For arbitrary seniority, the $Z_{v J}$ eigenvalues are found for the states of $J=J_{\max }=v(x-v+1) / 2$ and $J=J_{\max }-2$ to be

$$
\begin{aligned}
& Z_{v J_{\max }}=\frac{-v(x-v+1)}{(x+4)(x+3)(x+2)(x+1) x(x-1)(x-2)} \\
& \times\left(v x^{5}-11 v^{2} x^{4}+5 v x^{4}+12 x^{4}+45 v^{3} x^{3}-44 v^{2} x^{3}-48 v x^{3}+48 x^{3}-85 v^{4} x^{2}+135 v^{3} x^{2}\right. \\
& +102 v^{2} x^{2}-164 v x^{2}-24 x^{2}+75 v^{5} x-170 v^{4} x-85 v^{3} x+292 v^{2} x+12 v x-144 x-25 v^{6} \\
& \left.+75 v^{5}+25 v^{4}-175 v^{3}-24 v^{2}+124 v+48\right) \\
& Z_{v, J_{\max -2}}=\frac{-1}{(x+4)(x+3)(x+2)(x+1) x(x-1)(x-2)} \\
& \times\left(v^{2} x^{6}-12 v^{3} x^{5}+6 v^{2} x^{5}-36 v x^{5}+56 v^{4} x^{4}-60 v^{3} x^{4}+593 v^{2} x^{4}-180 v x^{4}+48 x^{4}-130 v^{5} x^{3}\right. \\
& +224 v^{4} x^{3}-2774 v^{3} x^{3}+2332 v^{2} x^{3}-432 v x^{3}+192 x^{3}+160 v^{6} x^{2}-390 v^{5} x^{2}+5588 v^{4} x^{2} \\
& -8082 v^{3} x^{2}+3376 v^{2} x^{2}-576 v x^{2}-336 x^{2}-100 v^{7} x+320 v^{6} x-5100 v^{5} x+10728 v^{4} x \\
& -7664 v^{3} x+2104 v^{2} x+48 v x-1056 x+25 v^{8}-100 v^{7}+1730 v^{6}-4840 v^{5}+5129 v^{4} \\
& \left.-2308 v^{3}+28 v^{2}+336 v+1152\right) \text {. }
\end{aligned}
$$

These are complicated but precise algebraic expressions. Algebraic expressions for the expectations of $\left(\hat{C}_{J} \otimes\right.$ $\left.\hat{C}_{J}\right)_{0}$ for other values of $J$ and other states could be even more complicated. Thus, although the above seniorityconserving models may be exactly solvable in principle for many multiplicity-free states, it is not known how to determine the energies of such states, in general, without resorting to numerical (and hence approximate) methods. This poses an interesting challenge.

Figure 1 shows the excitation energies of seniority $v=2$, and some $v=4$, states of some $N=50$ isotones computed for a seniority-conserving Hamiltonian that fits the $v=2, J=2,4$, and 8 states. It is interesting that, although the model predicts many more $v=4$ states than those of $J=J_{\max }=12$ and $J=J_{\max }-2=10$ for which we have analytical results, the $J=10$ and 12 states are the ones that are experimentally observed. The results provide much better fits to the experimental energies than those obtainable with the simple, fully solvable, $J=0$ pairing force model. In the $J=0$ pairing model all states of a nucleus of the same seniority occur at a common excitation energy and all $v=4$ states are at approximately twice the $v=2$ excitation energy.
The authors thank J.L. Wood and Ts. Dankova for valuable discussions and suggestions.

[1] A. G. Ushveridze, Quasi-Exactly Solvable Models in Quantum Mechanics (Institute of Physics, Bristol, 1994).

[2] Y. Alhassid and A. Leviatan, J. Phys. A 25, L1265 (1992); A. Leviatan, Phys. Rev. Lett. 77, 818 (1996).

[3] I. Talmi, Simple Models of Complex Nuclei (Harwood, Chur, Switzerland, 1991).

[4] G. Racah, Phys. Rev. 62, 438 (1942); 63, 367 (1943); 76, 1352 (1949).

[5] A. Arima and H. Kawarada, J. Phys. Soc. Jpn. 19, 1768 (1964); A. Arima and M. Ichimura, Prog. Theor. Phys. 36, 296 (1966).

[6] A. K. Kerman, Ann. Phys. (N.Y.) 12, 300 (1961); A.K. Kerman, R.D. Lawson, and M.H. MacFarlane, Phys. Rev. 124, 162 (1961); Nucl. Phys. 66, 80 (1965).

[7] B. H. Flowers, Proc. R. Soc. London A 212, 248 (1952).

[8] K. Helmers, Nucl. Phys. 23, 594 (1961).

[9] R. Howe, Lect. Appl. Math. 21, 179 (1985). 